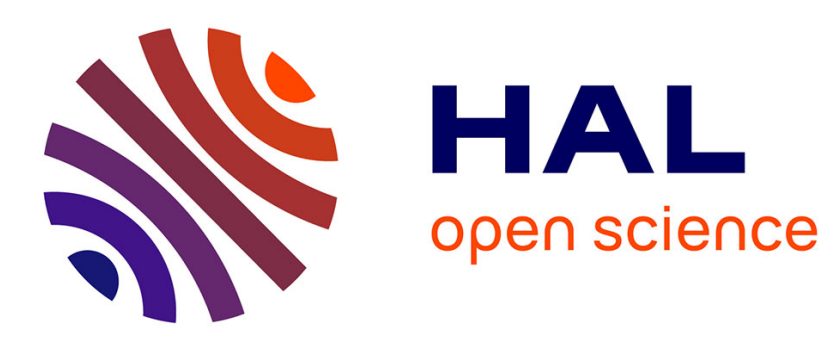

\title{
Parametric model of the BAW resonator phase-noise
}

\author{
M. Goryachev, S. Galliou
}

\section{To cite this version:}

M. Goryachev, S. Galliou. Parametric model of the BAW resonator phase-noise. Ultrasonics, 2011, 51 (8), pp.966-973. 10.1016/j.ultras.2011.05.012 . hal-00660544

\section{HAL Id: hal-00660544 https://hal.science/hal-00660544}

Submitted on 14 Apr 2021

HAL is a multi-disciplinary open access archive for the deposit and dissemination of scientific research documents, whether they are published or not. The documents may come from teaching and research institutions in France or abroad, or from public or private research centers.
L'archive ouverte pluridisciplinaire HAL, est destinée au dépôt et à la diffusion de documents scientifiques de niveau recherche, publiés ou non, émanant des établissements d'enseignement et de recherche français ou étrangers, des laboratoires publics ou privés. 


\title{
Parametric model of the BAW resonator phase-noise
}

\author{
Maxim Goryachev*, Serge Galliou \\ FEMTO-ST Institute, Frequency and Time Department, 26, Chemin de l'Epitaphe, Besançon 25000, France
}

Excepted for the very short terms the frequency stability of ultra-stable oscillators is mainly limited by the resonator noise. In this work we proposed a parametric model of the bulk acoustic wave (BAW) res-onator phase noise based on an equivalent circuit. This model explains phase noise generated by a BAW crystal from a point of view of parametric fluctuations and proves the $f^{-1}$ dependences of the crystal noise. The model performance is verified with simulation. Simulation results are compared to experimen-tal data and discussed. Comparison of three existing models is made.

\section{Introduction}

Experimental and theoretical investigation on the origins of phase noises is of great interest in recent years. The special attention is devoted to BAW resonators, because of their crucial role for many types of oscillators. Indeed, performance of ultra-stable BAW crystal oscillators (usually $5-10 \mathrm{MHz}$ ) in terms of frequency stability is limited by the resonator noise. Recent experimental researches on high quality bulk acoustic quartz resonators [1-4] provide a power spectral density (PSD) of the phase noise generated by quartz crystals. However, these results require further theoretical explanation. An explanation from a point of view of parameter fluctuations is given in this work.

There are two main approaches to a problem of BAW resonator phase noise. The first one (noise conversion method) is based on a resonator equivalent circuit and may be successfully used for phase noise PSD calculation of different oscillatory systems based on intrinsic noise sources [5,6]. The main advantage of this approach is that it enables us to study particular finite ranges of the system PSD and all types of noise without using the corresponding differential equations. This method is rather accurate and is in a good agreement with available measurements of that time.

The essence of the second approach (here and further called phase plane method) lies in the Laplace transform of the phase of a carrier and representation of the BAW crystal as a transfer function in a phase plane [9]. Though this method is common for different types of phase-locked loop (PLL) systems, it can also be applied to oscillatory system analysis. According to this approach phase

\footnotetext{
* Corresponding author. Tel.: +33 3814027 20; fax: +33 381885714

E-mail address: Maxim.Goryachev@femto-st.fr (M. Goryachev).
}

noise is regarded as additive flicker noise at the input of the resonator transfer function. No other noise sources and phenomena are considered. Nevertheless, this model may be sufficient for many types of system level analyses.

In this work we are trying to introduce another model which is also intended for system level analyses, but incorporates more noise phenomena than the described above models.

\section{Given data}

The model presented in this paper is based on recent measurements of different types of 5-10 $\mathrm{MHz}$ high quality quartz resonators from different manufacturers. These measurements have been performed by means of a home-made state-of-the-art interferometric measurement system. Some details about the test bench are given in [4] or [3], for example. The noise floor of the bench depends on the carrier power and, as a consequence, on the power dissipated inside the couple of quartz crystal resonators. It can vary according to the motional resistance of the resonator from $-143 \mathrm{dBc} / \mathrm{Hz}$, for a typical dissipated power of $20 \mu \mathrm{W}$, up to $-165 \mathrm{dBc} / \mathrm{Hz}$ for a typical dissipated power of $200 \mu \mathrm{W}$. Here and now it should be mentioned that experimental results do not exhibit any $f^{0}$ noise generated by both resonators, greater than the noise floor of the bench (so, it is also excluded from the model).

In addition, the amplitude-frequency effect have been observed on the resulting PSDs in the case of dissipated power typically greater than $75 \mu \mathrm{W}$ (in fact, it depends on the actual cut angles). This is described and discussed in [7]. A special attention must be paid to the measured cut-off frequency (the so-called Leeson's frequency), which, in some cases, may be not correct because of the aliasing effect of the asymmetric PSD on both sides of the carrier due to the quartz nonlinearities. 
Each resonator under test is temperature-controlled at its turnover temperature, in such a way that the thermal stability is about $2 \mu^{\circ} \mathrm{C}$ for measurement time of $1 \mathrm{~s}$ and about $20 \mu^{\circ} \mathrm{C}$ over $10 \mathrm{~s}$ [8]. Thus, the behaviour of $f^{-2}$-type noise PSD (see further) inside the resonators bandwidth is very limited or at least restricted to offset frequency lower than $0.01 \mathrm{~Hz}$.

A typical (about $90 \%$ of all measurements on various types of resonators) power spectral density plot of the quartz crystal phase noise is shown in Fig. 1. The curve shown in Fig. 1 consists of three parts: phase flicker $f^{-1}, f^{-3}$ and white $f^{0}$ noise areas. The latter is the noise floor of the measurement test bench and not a noise generated by the resonators under test.

Such a pattern has been explained with the phase plane model [9]. According to this model the phase noise at the output of the resonator results from a flicker phase noise at the input $\left(S_{\text {add }}\right)$ which is always present regardless the absence of the driving signal noise [9]. Thus, the power spectral density at the output of the resonator can be found in the following way:

$S_{\varphi}(f)=|\mathcal{H}(f)|^{2} S_{a d d}(f)$,

where $\mathcal{H}(f)$ is the transfer function of an ideal resonator in the phase plane and $f$ is the Fourier frequency. Since in absence of detuning signal this transfer function is a linear first order function, then a term $|\mathcal{H}(f)|^{2}$ has a spectral density with two regions: $f^{0}$ and $f^{-2}$. A multiplication of this term by $S_{\text {add }}$ which follows $f^{-1}$ law gives a power spectral density shown in Fig. 1 without the $f^{0}$ part. The same pattern may be easily obtained with the noise conversion method [6].

Nevertheless, in practice, for more modest resonators one can observe $f^{-2}$ slopes instead of $f^{-3}$ (see Fig. 2). This is the case of less than $10 \%$ of measured resonators and exclusively observed on just one type of resonators. This fact can be explained with the first approach based on fluctuations of the equivalent motional resistance, i.e. the noise conversion method, but not the phase plane model.

Here we consider a model based on parameter fluctuations of differential equations, which is close in nature to the second approach (representation of BAW resonator in phase space), but based on fluctuations of parameters of the electrical equivalent model, as in the first method.

\section{Parametric fluctuations}

Let us consider a simple $\pi$-network driven by an ideal sinusoid. A BAW crystal in this case can be represented by its electrical equivalent circuit (see Fig. 3).

In order to construct a rigorous model of parameter fluctuating system this equivalent circuit (Fig. 3) can be considered as a system, where motional inductance $L_{x}$ and capacitance $C_{x}$ vary with time in such a fast way that the Laplace transform of the signals be-

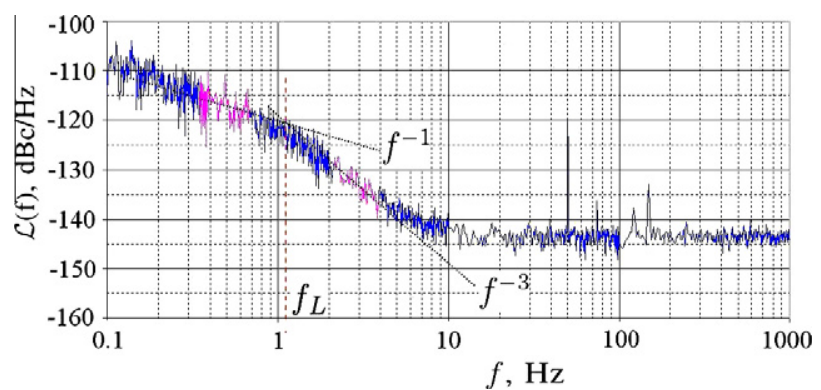

Fig. 1. Measured phase noise of a $5 \mathrm{MHz}$ high quality quartz crystal resonator. $f_{L}$ denotes the so-called Leeson frequency, i.e. a half of a resonator bandwidth. The dashed line is needed to identify the PSD value at $f=1 \mathrm{~Hz}$ from the carrier which is used to calculate the corresponding Allan deviation [2].

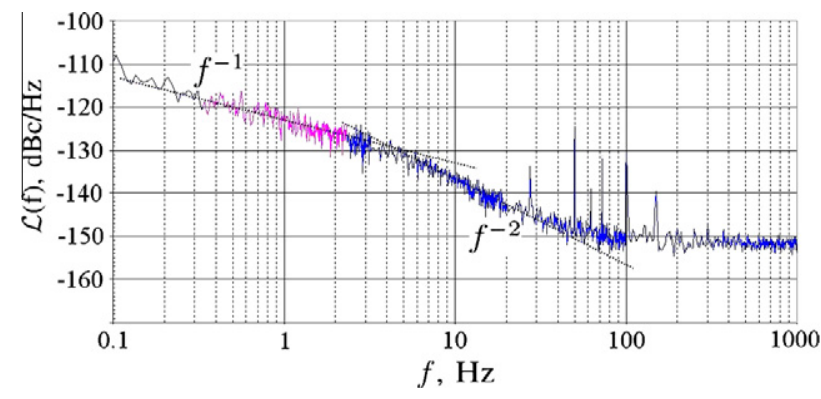

Fig. 2. Measured phase noise of a medium quality $10 \mathrm{MHz}$ crystal resonator. This behaviour has been observed just for one type of the tested resonators.

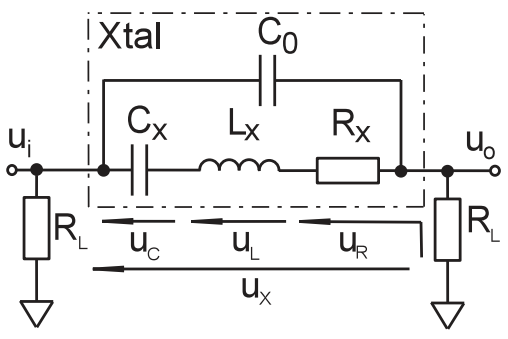

Fig. 3. Model of the BAW crystal as a part of the $\pi$-network.

comes irrelevant. Nevertheless, it is possible to derive ordinary differential equations (ODEs), which describe physical processes in such a system. The relations appear from the physical analysis of energy, which is stored in each of the components of the equivalent circuit. In fact, dynamic properties of a time-varying capacitance are associated with the time-varying electric charge and an inductance with magnetic flux [10]. Therefore, rigorously, the ODEs of the equivalent electrical circuit must be written and solved for the electric charge and/or magnetic flux, to fit physical processes.

This choice of fluctuating parameters is made, because an equivalent inductance and capacitance determine the position of the system natural frequency, which is believed to be a source of generated phase noise. Also, these two sources are separated since their influences on system parameters are different. For the simplicity of the following calculations the shunt capacitance $C_{0}$ Fig. 3 is neglected. Indeed, the absolute shift of the resonance frequency due to $C_{0}$ is estimated as follows:

$\Delta \omega \approx \omega_{x} \frac{R_{x}^{2} C_{0}}{L_{x}}$

where $\omega_{x}$ is the angular resonance frequency determined by the series motional branch $L_{x}, C_{x}, R_{x}$, where $R_{x}$ is the resonator equivalent series resistance. As an example, for a usual high quality quartz resonator, $R_{X}$ is less than $100 \mathrm{Ohms}, L_{x}$ is about $1 \mathrm{H}$ and $C_{0}$ is about $2 \mathrm{pF}$, that gives an absolute frequency shift of just about $10^{-8}$ of $\omega_{x}$. So, fluctuations of $C_{0}$ have $10^{8}$ lower impact on resonance frequency than that of $L_{x}$ and $C_{x}$. Moreover, the shunt capacitance is a function of the BAW resonator geometry and the relative permittivity. Both can fluctuate with temperature, inducing a $C_{0}$ relative sensitivity of a few $10^{-5}$ per Kelvin [11], but, in this work, the device under test is temperature-controlled. For the measurements resulting in Figs. 1 and 2 , temperature fluctuations of the tested resonators are typically lower than $2 \times 10^{-6} \mathrm{~K}$ over $1 \mathrm{~s}$ [8].

A time-varying parameter of the capacitor is its amount of electric charge $q$ stored for a given electric potential or the capacitance $C(t)=q(t) / u_{c}(t)$. The electrical current through the capacitor is a speed of change of its charge. From the following equation of the physical process in the capacitor can be derived: 
$i_{c}(t)=\frac{d q}{d t}=\frac{d q}{d u} \frac{d u_{c}}{d t}=C(t) \frac{d u_{c}}{d t}$,

where $u_{c}(t)$ is a capacitor voltage, $i_{c}(t)$ is a capacitor current.

In the same manner for a time-varying inductor the main parameter is the amount of magnetic flux $\xi$ produced for a given electric current $i_{L}$ in the inductance $\xi(t)=L(t) \cdot i_{L}(t)$. The inductor voltage is a derivative of the magnetic flux:

$u_{L}(t)=\frac{d \xi}{d t}=\frac{d}{d t} L(t) i_{L}(t)=\frac{d L(t)}{d t} i_{L}(t)+L(t) \frac{d i_{L}(t)}{d t}$.

Now, let us consider an equivalent circuit of the resonator which has time-varying components $L_{x}(t)=L_{x 0}+\Delta L_{x}(t)$ and $C_{x}(t)=C_{x 0}+\Delta C_{x}(t)$. So, the system governing equation can be written for a current through the crystal resonator $i_{x}$ as:

$\int_{-\infty}^{t} \frac{i_{x}(\tau)}{C_{x}(\tau)} d \tau+\frac{d L_{x}(t)}{d t} i_{x}(t)+\frac{d i_{x}(t)}{d t} L_{x}(t)+R_{x} i_{x}(t)+R_{L} i_{x}(t)=u_{i}(t)$

Substituting relation $u_{R}(t)=\left(R_{x}+R_{L}\right) i_{x}(t)$ into (4) and differentiating the result, the system equation can be expressed in terms of the total equivalent (motional resistance of the resonator $R_{x}$ and the load resistance $R_{L}$ ) resistance voltage drop $u_{R}(t)$ :

$\left.\ddot{u}_{R}+\dot{u}_{R} \frac{2 \dot{L}_{x}(t)+R_{x}+R_{L}}{L_{x}(t)}+u_{R} \frac{1}{C_{x}(t) L_{x}(t)}+\frac{\ddot{L}_{x}(t)}{L_{x}(t)}\right)=\dot{u}_{i} \frac{R_{x}+R_{L}}{L_{x}(t)}$,

so that the output voltage is linearly proportional to the total resistance voltage: $u_{o}=u_{R} \frac{R_{L}}{R_{L}+R_{x}}$.

Also, from this variant of description one can find an actual (dynamic) natural frequency and actual loaded quality factor of the crystal:

$\omega_{0}(t)=\sqrt{\frac{1}{C_{x}(t) L_{x}(t)}+\frac{\ddot{L}_{x}(t)}{L_{x}(t)}}, \quad Q(t)=\frac{L_{x}(t) \omega_{0}(t)}{2 \dot{L}_{x}(t)+R_{x}+R_{L}}$.

If the time dependences of $L_{x}(t)$ and $C_{x}(t)$ are known, Eq. (5) can be solved numerically by transforming the second order ODEs into a system of first order differential equations of the Cauchy form:

$\left\{\begin{array}{l}\dot{y}_{1}=y_{2} \\ \dot{y}_{2}=-\omega_{0}^{2}(t) y_{1}-2 \delta(t) y_{2}+2 k(t) x(t)\end{array}\right.$

where $y_{1}=u_{R}, x(t)=\dot{u}_{i}$ and

$\delta(t)=\frac{2 \dot{L}_{x}(t)+R}{2 L_{x}(t)}, \quad k(t)=\frac{R}{2 L_{x}(t)}$.

For the sake of simplicity all following calculations are made for the total equivalent resistance $\left(R=R_{L}+R_{x}\right)$. However, this task is very time-consuming. The point is that the frequency of the input signal has to be in the vicinity of the resonance frequency of the resonator crystal, i.e. the period of change is about $10^{-7} \mathrm{~s}$. But in the same time fluctuations of the parameters of the system are very slow. Here we are interested in the processes with time constants of tens and hundreds of seconds. Thus, a number of time steps for modelling of such a system is incredibly large. In order to avoid this drawback the method of averaging [10,12-14] can be used.

\section{System solution with averaging method}

Let the input signal of the $\pi$-network is described in the form of an ideal cosinusoid:

$u_{i}(t)=u_{a} \cos (\omega t+\varphi)$,

where $\omega$ is a constant angular frequency. Then the signal $x(t)$ is a derivative of the $u_{i}(t)$ : $x(t)=\dot{u}_{i}(t)=-u_{a} \omega \sin (\omega t+\varphi)=-u_{a} \omega \cos \left(\omega t+\varphi-\frac{\pi}{2}\right)$.

A state vector $\boldsymbol{Y}=\left[y_{1}(t), y_{2}(t)\right]^{T}$ shows the position of the system at the present moment $t$ in the phase plane. These are Cartesian (rectangular) coordinates of the point. In this case both coordinates have the same rate of change. But as it is known from the control system theory a system in the phase plane can be represented by an infinite number of ways, i.e. state coordinates. For example, in the polar coordinates the system can be described by a length $m(t)$ (magnitude) and an angle $\psi(t)$ (phase) of a vector drawn from the point of origin to the current state: $\boldsymbol{M}=[m(t), \psi(t)]^{T}$. For the given system the transition from the Cartesian coordinates to the polar ones is done with the following expressions:

$y_{1}(t)=m(t) \cos (\omega t+\phi(t))=m(t) \cos (\psi(t))$,

$y_{2}(t)=\frac{d y_{1}(t)}{d t} \approx-m(t) \omega \sin (\psi(t))$.

The coordinate $y_{2}(t)$ is found for the case when the length of the vector $m(t)$ and the phase fluctuation $\phi(t)$ change much slower than the phase $\omega t$. In this variant $m(t)$ and $\phi(t)$ change with the rate of parameters of the equivalent circuit $L_{x}(t)$ and $C_{x}(t)$, and the phase $\omega t$ changes with the rate of the input signal.

Substituting expressions (9) to the system in Cartesian coordinates (see Eq. (7)), reducing equal term in left-hand and right-hand sides and dividing the second equation by $-\omega$, the following system in polar coordinates is derived:

$$
\left\{\begin{array}{l}
\dot{m} \cos \psi-m \dot{\phi} \sin \psi=0, \\
\dot{m} \sin \psi+m \dot{\phi} \cos \psi=-m \omega \cos \psi+\frac{\omega_{0}^{2}}{\omega} m \cos \psi-2 \delta m \sin \psi \\
\quad+2 \frac{k u_{a}}{m} \sin (\omega t) .
\end{array}\right.
$$

This system can be transformed to the Cauchy form for slowly varying variables $m$ and $\phi$. To do this, the first equation of system (10) has to be multiplied by $\cos \psi$ and the second one by $\sin \psi$. After summating the results of these multiplications and taking into account the Pythagorean trigonometric identity for functions of $\psi$, the first equation of system (11) is obtained. After that the procedure needs to be repeated. This time the first equation has to be multiplied by $-\sin \psi$, and the second one by $\cos \psi$. After summation, the second equation of (11) is derived.

$$
\left\{\begin{aligned}
\dot{m}= & -m \omega \sin \psi \cos \psi+\frac{\omega_{0}^{2} m}{\omega} \sin \psi \cos \psi-2 \delta m \sin ^{2} \psi \\
& +2 k u_{a} \sin (\omega t) \sin \psi, \\
\dot{\phi}= & -\omega \cos ^{2} \psi+\frac{\omega_{0}^{2}}{\omega} \cos ^{2} \psi-2 \delta \sin \psi \cos \psi+2 \frac{k u_{a}}{m} \sin (\omega t) \cos \psi .
\end{aligned}\right.
$$

System (11) still depends on the fast component of the phase. So, in order to spare from this constituent both parts of both equations have to be integrated over one period of the input signal $T=\frac{2 \pi}{\omega}$, or just $2 \pi$ for the variable $\psi$.

The following result is obtained with an assumption that the length $m$ and the phase $\phi$ do not change during one period of the input signal. In case of parameter fluctuations of BAW resonators, this assumption holds true with good precision. Thus, swapping derivation and integration (taking in mind that $\dot{\psi} \approx \omega$, so $\phi$ and $\psi$ are independent) we obtain a system for length $M$ and phase $\Phi$ of an average vector, which describes a system state for one period of the input signal. 


$$
\left\{\begin{aligned}
2 \pi \dot{M}= & -M \omega \int_{0}^{2 \pi} \sin \psi \cos \psi d \psi+\frac{\omega_{0}^{2} M}{\omega} \int_{0}^{2 \pi} \sin \psi \cos \psi d \psi \\
& -2 \delta M \int_{0}^{2 \pi} \sin ^{2} \psi d \psi+2 k u_{a} \int_{0}^{2 \pi} \sin (\omega t) \sin \psi d \psi, \\
2 \pi \dot{\Phi}= & -\omega \int_{0}^{2 \pi} \cos ^{2} \psi d \psi+\frac{\omega_{0}^{2}}{\omega} \int_{0}^{2 \pi} \cos ^{2} \psi d \psi \\
& -2 \delta \int_{0}^{2 \pi} \sin \psi \cos \psi d \psi+\frac{2 k u_{a}}{M} \int_{0}^{2 \pi} \sin (\omega t) \cos \psi d \psi
\end{aligned}\right.
$$

Each of three first integrals of both equations of system (12) is trivial. The last integrals are found taking into account the definition $\psi=\omega t+\phi$, which means that $\omega t=\psi-\phi$. And, so it can be found using the well-known trigonometric identities for the sinus and the cosinus of the difference:

$$
\begin{gathered}
\int_{0}^{2 \pi} \sin (\psi-\phi) \sin \psi d \psi=\int_{0}^{2 \pi} \sin ^{2} \psi \cos \phi d \psi \\
-\int_{0}^{2 \pi} \cos \psi \sin \phi \sin \psi d \psi=\pi \cos \Phi .
\end{gathered}
$$

In the same way the last integral of the second equation of (12) gives:

$$
\begin{aligned}
\int_{0}^{2 \pi} \sin (\psi-\phi) \cos \psi d \psi & =\int_{0}^{2 \pi} \sin \psi \cos \phi \cos \psi d \psi \\
-\int_{0}^{2 \pi} \cos ^{2} \psi \sin \phi d \psi & =-\pi \sin \Phi .
\end{aligned}
$$

And, finally, the system of differential equations for averaged values of length and phase of the vector can be written in the following way:

$$
\left\{\begin{array}{l}
\dot{M}=-\delta(t) M+K(t) u_{a} \cos \Phi, \\
\dot{\Phi}=\Omega(t)-K(t) \frac{u_{a}}{M} \sin \Phi,
\end{array}\right.
$$

where $\Omega=\frac{\omega_{0}^{2}(t)-\omega^{2}}{2 \omega}$ is a detuning frequency. And $M$ and $\Phi$ are average values of $m(t), \phi(t)$ for one period of the input signal.

In order to find equilibrium points the left parts of the system (13) have to be set equal to zeros:

$$
\left\{\begin{array}{l}
\delta(t) M=K(t) u_{a} \cos \Phi, \\
\Omega(t) M=K(t) u_{a} \sin \Phi .
\end{array}\right.
$$

If the system is time-invariant and the frequency of the input signal corresponds to the natural frequency of the resonator (i.e. $\Omega=0$ ), then the second equation of system (14) gives solutions for $\Phi$ in the form $\Phi=\pi n$, where $n \in \mathbb{Z}$. This solution of the first equation gives two possible variants of the first one: $M= \pm u_{a}$. A negative variant of the solution does not make physical sense. Moreover only one solution (on case of $n=0$ ) can be explained in the physical way. This result is a solution, where the BAW crystal is in the resonance, i.e. it does not give a phase shift to the input signal $(\Phi=0)$ and the input voltage equals to the total resistance voltage drop $\left(M=u_{a}\right)$. The point $\left(u_{a}, 0\right)$ in $(M, \Phi)$ space is the stable equilibrium point of system (13).

Figs. 4 and 5 show amplitude $M$ and phase $\Phi$ fluctuations correspondingly produced by some random fluctuations of $L_{x}(t)$ and $C_{x}(t)$ and simulated with system (13), where the input amplitude is $u_{a}=1 \mathrm{~V}$.

Fig. 4 shows that $M=u_{a}=1 \mathrm{~V}$ is a coordinate of the stable equilibrium point of system (13). From the physical point of view, $M$ cannot exceed $u_{a}$. The same result is obtained with the model. When $M=u_{a}$ the BAW crystal is exactly in the resonance. In all other points inductor and capacitor impedances are not compensated and the resonator is not working in the resonance mode.

Fig. 5 shows that the output phase fluctuations are observed around another coordinate of the equilibrium point $\Phi=0$. When $\Phi=0$, the system is in the resonance mode, when impedances of the capacitor $C_{x}$ and the inductance $L_{x}$ are compensated and the crystal does not give a phase shift between input and output signals.
So, system of differential Eq. (13) can be used to find phase and amplitude fluctuations for given fluctuations of equivalent capacitance $C_{x}$ and inductance $L_{x}$, introduced by fluctuating detuning frequency $\Omega$ and coefficients $\delta$ and $K$, and vice versa. In both cases a system of differential equations has to be solved.

\section{Numerical simulation}

If the PSDs of parameter fluctuations are known, then the numerical method of finding the generated phase noise PSD can be represented with the following steps:

1. generating a set of signals (fluctuations) in the time domain with a PSD corresponding to the known PSD of parameter fluctuations (several signals with the same PSD are needed to verify if the solution is the same for the whole class of fluctuations, in other words, it does not depend on the exact form of these fluctuations);

2. substitution of each signal into system (13);

3. solving system (13) for $M$ and $\Phi$ for each signal from the set;

4. finding the PSD for time-domain signals obtained in the previous steps and averaging of results for the given set. If all signals from the set produce the same result, then this result is considered as correct. Finding the confidence range.

To generate random fluctuations (random phase for each Fourier frequency) for the given PSD the following algorithm can be used:

1. to take the square root of the absolute value of the given PSD for each data point to get a magnitude spectral density: $|X(f)|=\sqrt{S_{x}(f)}$;

2. to generate random (uniform probability density) phase in frequency domain: $\Psi(f)=2 \pi n(f)$, where $n(f)$ is a random number generated for each frequency

3. to take the Inverse Fourier Transform of the random spectrum: $\Delta L_{x}(t)=\mathcal{F}^{-1}(|X(f)| \cdot \exp (j \Psi(f)))$, where $\mathcal{F}^{-1}(\cdot)$ is the Inverse Fourier Transform.

Note should be taken that if the PSD of the phase fluctuations is limited by the frequency which is less than the sampling frequency of the system, then the generated signal is differentiable. And differentiability is a mandatory requirement for the fluctuations of system parameters.

Calculations have been made (see Fig. 6) for a high quality quartz crystal (BVA-type, SC-cut) with the following parameters: resonance frequency $f_{0}=10 \mathrm{~Hz}$ ( $C$ mode, 3 rd overtone), motional resistance $R_{x}=90.12 \mathrm{Ohm}$, average motional inductance $L_{x}=1.79 \mathrm{H}$, average motional capacitance $C_{x}=141 \mathrm{aF}$. So, for this crystal resonator the unloaded $Q$ is approximately $1.25 \times 10^{6}$. Numerical simulations show that in order to obtain a result in accordance with experimental observations (i.e. $f^{-1}$ and $f^{-3}$ regions) the original PSD of $L_{x}$ and $C_{x}$ fluctuations must follow $f^{-1}$ law.

As expected, if fluctuations of the equivalent inductance is a random ergodic process with a $f^{-1}$ power spectral density, then the resulting PSD of average phase fluctuations has two regions, where the power spectrum is proportional to $f^{-1}$ and $f^{-3}$ (see Fig. 6). Fig. 6 shows three PSD curves for three different levels of $L_{x}$ fluctuations: $-45 \mathrm{dBc} / \mathrm{Hz}$ (curve 1), $-50 \mathrm{dBc} / \mathrm{Hz}$ (curve 2), $-55 \mathrm{dBc} / \mathrm{Hz}$ (curve 3 ) at $1 \mathrm{~Hz}$. This result is obtained for all generated signals of parameter fluctuations from the inital set. The inductance fluctuations $\Delta L_{x}(t)$ also result in fluctuations of the system natural frequency, as it was stated earlier.

The Leeson frequency of the resonator under study is a half of its bandwidth $f_{L} \approx \frac{f_{0}}{2 Q_{L}} \approx 3.9 \mathrm{~Hz}$. However, this formula cannot be regarded as precise, because a loaded quality factor $Q_{L}$ is a function 


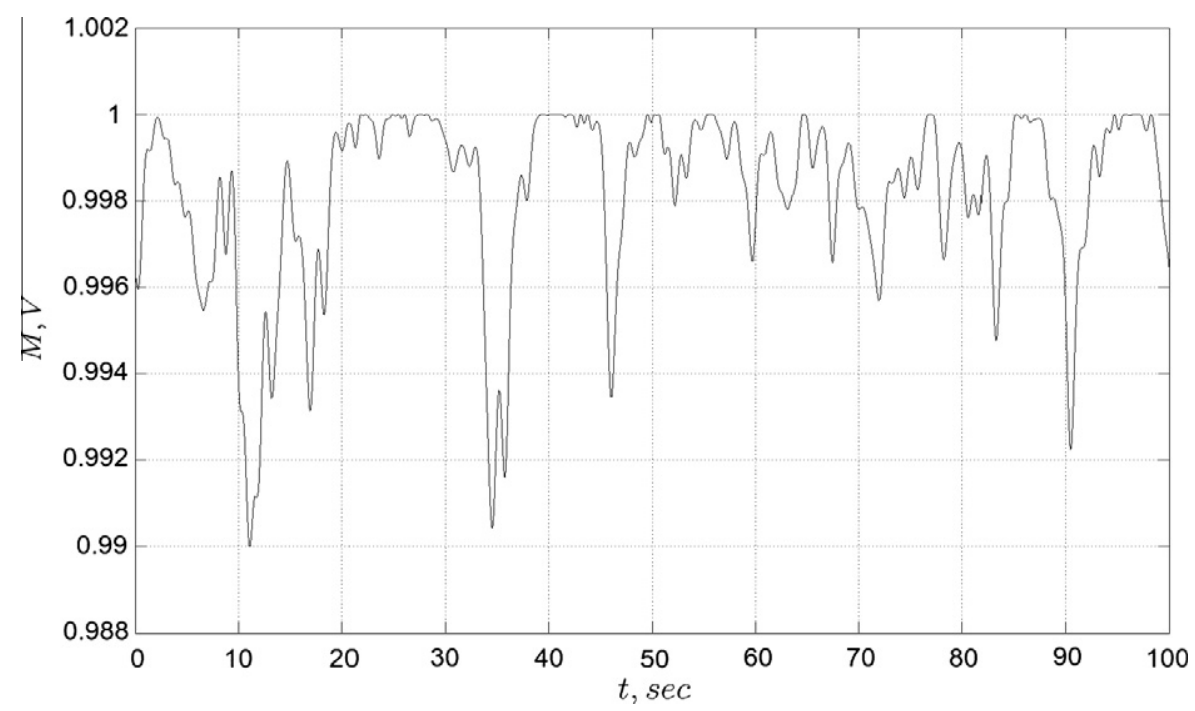

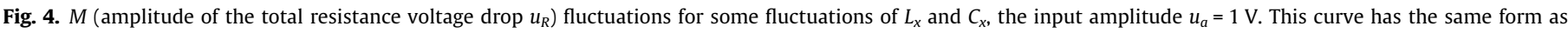
output amplitude (see (6)).

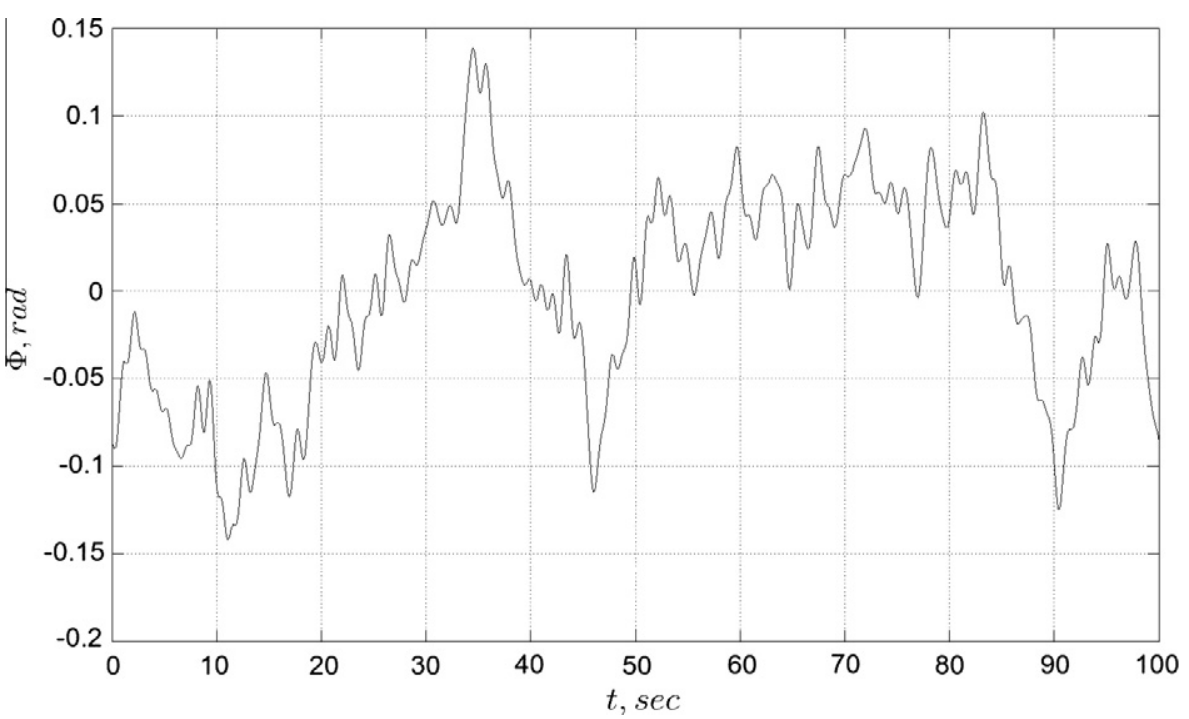

Fig. 5. $\Phi$ (phase of the motional resistance signal and the output signal) fluctuations for some fluctuations of $L_{x}$ and $C_{x}$.

of time according to expression (6). However, Fig. 6 shows approximately the same result as a border frequency between $f^{-1}$ and $f^{-3}$ regions.

It can be seen from Fig. 6 that for smaller values of input fluctuations the resulting PSD curves have more distortions, thus, the slope $f^{-3}$ may be mistakenly regarded as $f^{-2}$. This fact is a result of numerical limitations of the used algorithm for direct and inverse Fourier Transforms (limitation of data types). But it is evaluated that in order to achieve the level of about $-135 \mathrm{dBc} / \mathrm{Hz}$ at $1 \mathrm{~Hz}$, the level of $L_{x}$ fluctuations has to be approximately $-56 \mathrm{dBc} / \mathrm{Hz}$ at the same frequency.

Also it has to be mentioned that for other spectral laws of input parameter fluctuations $\left(f^{0}, f^{-2}\right.$ or any other) the output fluctuations have different (from those shown in Fig. 6) PSD configurations.

\section{Influence of the equivalent resistance noise}

The model described above does not take into account flicker and thermal noise of the equivalent series and load resistances.
These types of noises can be easily introduced into the model. In fact, the result system (13) remains valid even in this case, but parameters $\omega_{0}^{2}, \delta$ and $K$ have to be extended:

$\omega_{0}^{2}=\frac{1}{C_{x} L_{x}}-\frac{2 \dot{R}}{R}\left[\frac{\dot{L}_{x}}{L_{x}}-\frac{\dot{R}}{R}\right]-\left[\frac{\ddot{R}}{R}-\frac{\ddot{L}_{x}}{L_{x}}\right], \quad \delta=\frac{2 \dot{L}_{x}+R}{2 L_{x}}-\frac{\dot{R}}{2 R}$,

$K=\frac{R}{2 L_{x}}$.

These three parameters represent system noise sources, amongst which $\omega_{0}^{2}$ is dominant for a considered frequency interval. This parameter represent a squared natural frequency of a resonator and its fluctuations are equivalent to frequency fluctuations of the input signal.

System parameter $\omega_{0}^{2}$ consists of three terms. The first term $-\frac{1}{C_{C} L_{x}}$ has a spectrum slope $f^{-1}$ (when $C_{x}$ and $L_{x}$ fluctuate according to $f^{C_{x} L_{x}}$ PSD) and produces clear pattern of $f^{-1}$ and $f^{-3}$ noise PSDs slopes at the resonator output. The second term has a PSD slope of $f^{0}$ for all frequencies of the spectrum, if $R$ noise has also flicker spectrum. By, applying the same rule as to the first term, the 


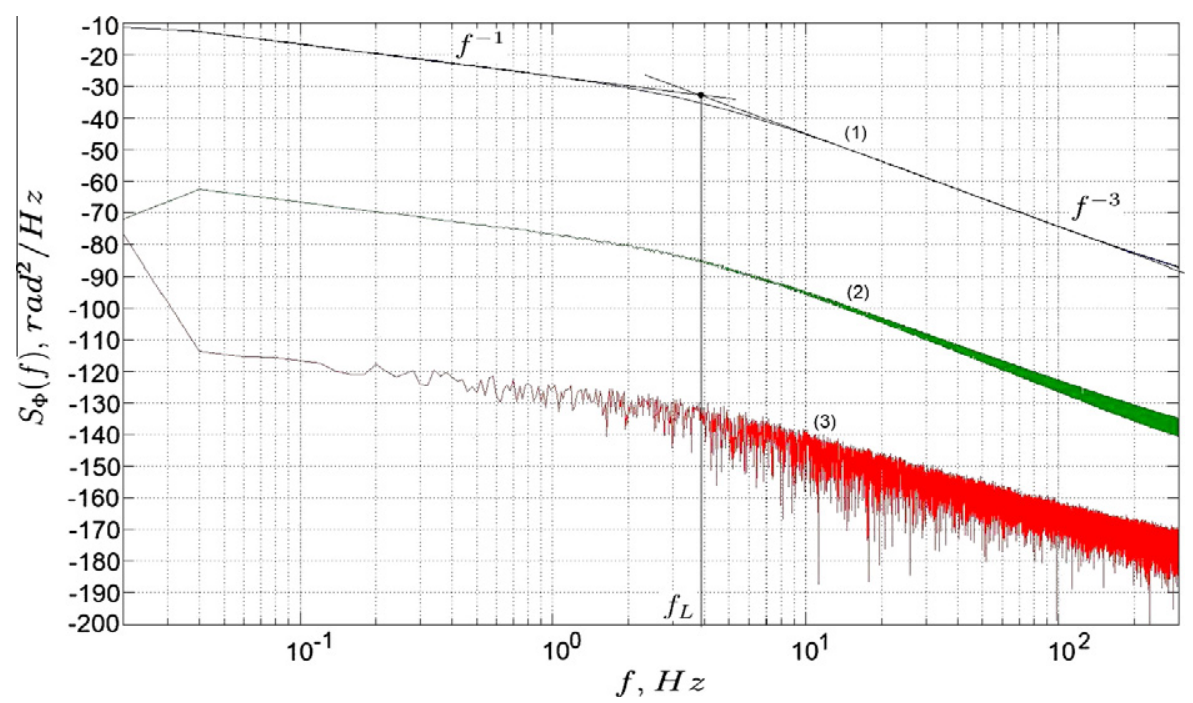

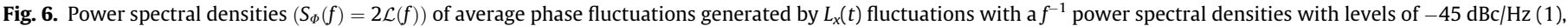
$-50 \mathrm{dBc} / \mathrm{Hz}(2)$, and $-55 \mathrm{dBc} / \mathrm{Hz}(3)$ at $1 \mathrm{~Hz}$.

resulting PSD of the system, when only this term is present, consists of $f^{0}$ and $f^{-2}$. The third term also results in white noise of the equivalent resonance frequency $\omega_{0}$, and so produces $f^{0}$ and $f^{-2}$ spectral laws.

Fig. 7 presents three PSDs, generated separately by the first (curve (1)), second (curve (2)) and third terms (curve (3)) of $\omega_{0}^{2}$. As it is shown in this figure, $f^{-2}$ can be seen instead of $f^{-3}$ for some resonators, if phase noise generated by the second and third terms of $\omega_{0}^{2}$ dominate in this region. Thus, this effect depends on resonator parameter (static values of $C_{x}, L_{x}$ and $R$ ), as well as noise levels of these parameters. So, it is not surprising that this effect is present for some types of crystal resonators.

\section{Link between the phase plane model and the present approach}

System (13) shows a dynamical link between fluctuations of the output signal in form of amplitude $M$ and phase $\Phi$, and fluctuations of the model parameters (equivalent inductance $L_{x}$ and equivalent capacitance $C_{x}$ ). Such a system represents time-varying system, where excitation is introduced with parameter fluctuations. A similar relationship may be written for a case of time-invariant additive model of the BAW crystal phase noise, when excitation is introduced with a phase noise of the input signal:

$u_{i}(t)=u_{a} \cos (\omega t+\theta(t))$,

where $\theta$ is an additive phase noise. When a signal with an addive phase noise is applied to the $\pi$-network, it is filtrated in the phase plane. Following the same procedure as in the case of the parametric model, another system in terms of output averaged amplitude and phase $(M, \Phi)$ may be derived (16):

$$
\left\{\begin{array}{l}
\dot{M}=-\delta M+k u_{a} \frac{(\omega+\dot{\Theta})}{\omega} \cos (\Phi-\Theta), \\
\dot{\Phi}=\Omega+k \frac{u_{a}}{M} \frac{(\omega+\dot{\Theta})}{\omega} \sin (\Phi-\Theta)
\end{array}\right.
$$

where $\Theta$ is the average (for one period of the input signal) value of input phase fluctuations $\theta(t)$, and $\Omega, \delta$ and $k$ are time-invariant parameters.

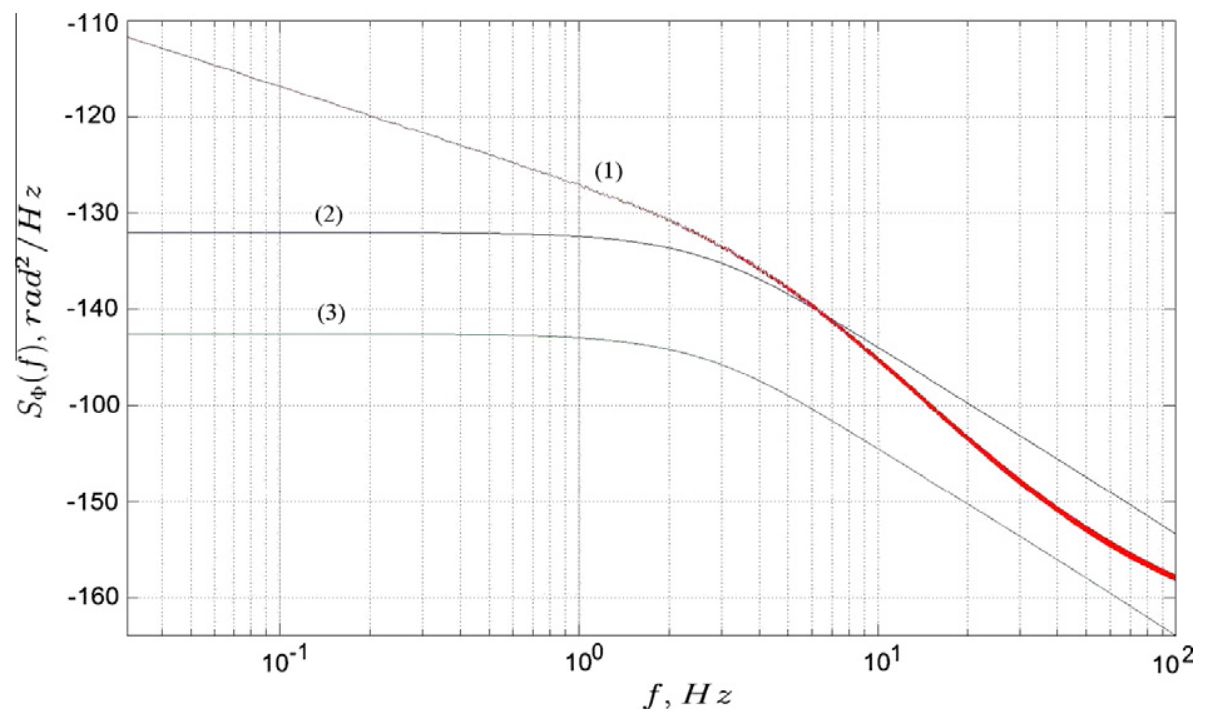

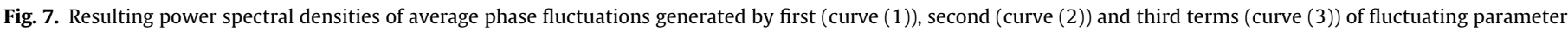
$\omega_{0}^{2}(15)$, when $L_{x}, C_{x}$ and $R_{x}$ disturbances has flicker noise PSD. The levels of applied noise are arbitrary. 
If the input phase noise PSD follows $f^{-1}$ law, then simulation of this system with the method described above showed the same results as simulation of system (13), i.e. the output phase noise PSD has $f^{-1}$ and $f^{-2}$ regions with Leeson frequency of about $3.9 \mathrm{~Hz}$. This fact proves that a BAW crystal inside the $\pi$-network acts as a first order filter in the phase plane. In fact, both systems ((13) and (16)) represent the same nonlinear dynamics, but with different inputs of excitation.

Assuming the following simplifications:

$1+\frac{\dot{\Theta}}{\omega} \approx 1, \quad \frac{\omega^{2}-\omega_{0}^{2}}{2 \omega} \approx \omega-\omega_{0}=-\Omega$,

the same system of equations may be used to find the resonator transfer functions in the phase space:

$\mathcal{H}(s)=\frac{\Phi(s)}{\Theta(s)}=\frac{\delta s+\delta^{2}+\Omega^{2}}{s^{2}+2 \delta s+\delta^{2}+\Omega^{2}}$.

The transfer function corresponds to the phase plane model obtained with another heuristic approaches [4,9]. In fact, the latter is a special case of the present model with the following main simplifications: (1) fluctuations of $\delta$ and $k$ are discarded (see (13)); (2) simplifications described in (17) are used; and (3) the second and third terms of parameter $\omega_{0}^{2}$ (see (15)) are excluded.

Moreover, if both input amplitude and phase noise and inner parametric noise are of interest for some reasons the model may be extended:

$$
\left\{\begin{array}{l}
\dot{M}=-\delta(t) M+K(t)\left[-\frac{\dot{u}_{a}}{\omega} \sin (\Phi-\Theta)+u_{a}\left(1+\frac{\dot{\Theta}}{\omega}\right) \cos (\Phi-\Theta)\right] \\
\dot{\Phi}=\Omega(t)+K(t) \frac{1}{M}\left[-\frac{\dot{u}_{a}}{\omega} \cos (\Phi-\Theta)-u_{a}\left(1+\frac{\dot{\Theta}}{\omega}\right) \sin (\Phi-\Theta)\right] .
\end{array}\right.
$$

This system of differential equations represents a Multiple Input Multiple Output (MIMO) time-varying transfer function for amplitude and phase noises, which is the most general case of resonator representation in amplitude-phase space.

\section{Comparison of the noise conversion model and the present approach}

Here two cases are distinguished: within and beyond half bandwidth, which are separated with Leeson's frequency $f_{L}$.

Within half bandwidth both models are in full correspondence. The shape of the PSD is determined by the effect of motional inductance and capacitance flicker noise. Futhermore, according to all three approaches, the level of the resulting flicker noise is proportional to the squared loaded quality factor $\left(Q_{L}^{2}\right)$, in terms of $S_{\Phi}(f)=2 \mathcal{L}(f)$.

Beyond the half bandwidth, models are in contrast to each other. First, let's consider results predicted by the noise conversion method. According to the results obtained in [6] the PSD of the resonator phase noise may be formed by the following noise sources: motional inductance and capacitance flicker noise $\left(f^{-5}\right)$, resonator additive thermal noise $\left(f^{-2}\right)$, motional resistance flicker noise $\left(f^{-3}\right)$, load noise $\left(f^{0}\right)$. So, as it has been concluded in this paper: the PSD slope beyond the half bandwidth can vary from $f^{-3}$ to $f^{-2}$ as a result of losses additive thermal and flicker noises. For more information see [6].

The contradictory points of the present model and the noise convertion method are:

1. motional resistance flicker noise according to the simulations presented here may produce $f^{-2}$ for certain resonators, but not $f^{-3}$ as in [6];
2. as it has been shown both analytically and numerically, the motional inductance and capacitance flicker noise results in $f^{-3}$ phase noise slope at the output (but not $f^{-5}$ ), which is confirmed by experiments in $90 \%$ of all cases. Also, $L_{x}$ and $C_{x}$ are the only sources of $f^{-3}$ noise according to the previous point;

3 . the motional resistance additive thermal noise cannot be considered as a noise source for $f^{-2}$ phase noise. Indeed, in accordance with [6] the level of the noise at the output can be calculated as follows:

$$
\mathcal{L}(f)=\frac{2 k T}{P} \frac{f_{L}^{2}}{f^{2}},
$$

where $k$ is Boltzmann's constant, $T=350 \mathrm{~K}, P=20 \mu \mathrm{W}$ is the carrier power, and $f_{L} \approx 4.5 \mathrm{~Hz}$ for the case shown in Fig. 2. With this data one may obtain with $(20)-153 \mathrm{dBc} / \mathrm{Hz}$ at $f=f_{L}$, which is about the same level as the measurement bench noise. And, the actual measured $f^{-2}$ noise is about $25 \mathrm{dBc} / \mathrm{Hz}$ higher.

4. $f^{0}$ slope of the resulting PSD has been discarded, since it cannot be seen with the present measurement equipment, which is the main source of $f^{0}$ noise in Figs. 1 and 2.

Since the results of two approaches contradict each other, further scientific discussions and measurements are badly needed for finding the actual sources of the resonator noise.

\section{Conclusion}

It can be concluded from the results of the previous numerical experiments, that most common patterns of the phase noise at the output of the BAW resonator (See Figs. 1 and 2) can be regarded as a result of its dynamics $L_{x}$ (as well as, $C_{x}$ and $R_{x}$ ) fluctuations with $f^{-1}$ spectrum. Inside the resonator half bandwidth the resulting flicker phase noise is determined by $f^{-1}$ fluctuations of $L_{x}$ and $C_{x}$. Outside the half bandwidth the phase noise can be formed by pairs $C_{x}-L_{x}$ with the result of $f^{-3}$ or $L_{x}-R_{x}$ with the result of $f^{-2}$. The first case is observed experimentally in $90 \%$ of measurements. And the remaining $10 \%$ have been observed just on one type of resonators.

The simulation results testify to the validity of the proposed way of resonator phase noise modelling. Contrary to ordinary phase space method the approach proposed in this paper is based on consideration of the true phase noise origin, but not a virtual additive phase noise. This origin lies in parameter fluctuations of the BAW crystal due to fluctuations of temperature, pressure, etc. So, the proposed method confirms with simulation a guess that phase noise generated by a BAW crystal is a result of natural frequency fluctuations. The presented model is more general and may incorporate link between magnitude and phase noises in the cases of parametric noise, input additive noise and both (19). Nevertheless, the main difficulty is connected with the fact that the presented approach has not been reduced to simple engineering solutions. Instead one can use the ordinary phase space model as a simplified solution.

In the same time the results obtained with this approach contradict the results of noise convertion method for the phase noise beyond the half bandwidth. This leads us to futher discussions on BAW resonator phase noise origins.

\section{Acknowledgement}

This work is supported by Conseil Régional de Franche-Comté (Convention No. 2008C_16215). The authors wish to thank them for the material support as well as the staff of the Time and Frequency Department of FEMTO-ST Institute, Besançon, France for their help and fruitful discussions, especially Xavier Vacheret for the measurements. 


\section{References}

[1] S. Galliou, F. Sthal, X. Vacheret, R. Brendel, P. Salzenstein, E. Rubiola, G. Cibiel, A program to analyze the origin of noise in ultra-stable quartz crystal resonators, in: Proceedings of IEEE International Frequency Control Symposium/European Frequency and Time Forum, 2007, pp. 1176-1181.

[2] F. Sthal, S. Galliou, J. Imbaud, X. Vacheret, P. Salzenstein, E. Rubiola, G. Cibiel, About quartz crystal resonator noise: recent study, in: Proceedings of the 20th International Conference on Noise and Fluct, 2007, pp. 607-610.

[3] F. Sthal, M. Mourey, F. Marionnet, W.F. Walls, Phase noise measurements of $10 \mathrm{MHz}$ BVA quartz crystal resonator, IEEE Trans. Ultrason. Ferroelec. Freq Control 47 (2000) 369-373.

[4] E. Rubiola, J. Groslambert, M. Brunet, V. Giordano, Flicker noise measurement of HF quartz resonators, IEEE Trans. Ultrason. Ferroelec. Freq. Control 47 (2000) 361-368.

[5] Y.S. Shmaliy, Conversion of $1 / \mathrm{f}$ fluctuations in crystal resonator within an inter resonance gap, IEEE Trans. UFFC 46 (1999) 61-71.

[6] Y.S. Shmaliy, The noise conversion method for oscillatory systems, IEEE Trans. UFFC 51 (2004) 948-956.
[7] F. Sthal, S. Galliou, J. Imbaud, X. Vacheret, P. Salzenstein, G. Cibiel, E. Rubiola, The effect of power-drive level on the calibration of the bridge instrument for the measurement of the quartz stability, in: Proceedings to the IEEE International Frequency Control Symposium/European Frequency and Time Forum, 2009, pp. 487-491.

[8] F. Sthal, S. Galliou, P. Abbe, N. Franquet, X. Vacheret, P. Salzenstein, E. Rubiola, G. Cibiel, Thermal characterization of crystal ovens used in phase noise measurement system, in: Proceedings of the IEEE International Frequency Control Symposium, 2006, pp. 736-739.

[9] E. Rubiola, Phase Noise and Frequency Stability in Oscillators, Cambridge University Press, 2008.

[10] Y.S. Shmaliy, Continuous-Time Systems, Springer, Dordrecht, 2007.

[11] R.W. Ward, The constants of alpha quartz, in: Proceedings Piezoelec Devisus Conference and Exhibit, 1992.

[12] A.I. Chulichkov, Mathematical Models of Nonlinear Dynamics, second ed., FIZMATLIT, Moscow, 2003.

[13] N.M. Krylov, N.N. Bogolyubov, Introduction to Non-Linear Mechanics, Princeton University Press, Princeton, 1947.

[14] A.H. Nayfeh, D.T. Mook, Nonlinear Oscillations, John Wiley\& Sons, USA, Canada, 1995. 